\title{
Free-stream turbine start-up under load
}

\author{
Svyatoslav Khorobrov ${ }^{1, *}$, Daria Nemova ${ }^{1}$, Daniil Gorbatov ${ }^{1}$, Vladimir Kalaev², and Eugene Kotov ${ }^{1}$ \\ ${ }^{1}$ Peter the Great St. Petersburg Polytechnic University, St. Petersburg, Russia \\ ${ }^{2}$ LLC "GTR", St. Petersburg, Russia
}

\begin{abstract}
Free-stream turbine is an alternative to classical high-head turbines. On one hand, it produces less power, on the other hand, it requires less ser-vice and construction and might be an eco-friendlier option. We have modelled the start-up of a free-stream turbine, which consists of a wheel and a generator, using CFD methods. Unsteady RANS method with k- $\omega$ SST model was employed to describe turbulence. We have used finite-volume solver Ansys FLUENT on unstructured mesh. Moving mesh approach was applied for the wheel rotation. We have based the blade sys-tem design on several simple assumptions. Wheel inertia and generator load were taken into account through simple moment equations. Velocity fields during different moments of time were obtained. We have obtained both momentum and power of the wheel curves over time and reviewed cases with generator presence and absence. Angular velocity curves comparison over time is presented for two cases.
\end{abstract}

\section{Introduction}

Polytechnic University is known for it's turbomachinery researches [1-3].More than $60 \%$ of electricity power in Russia is produced by thermal power station, according to report from UES of Russia. Only $20 \%$ of electricity power is produced by hydro power plants, and less, than $1 \%$ is produced with solar and wind power stations [4]. Climate conditions wouldn't allow to construct solar and wind power stations all over the country, so hydro power plants seem to be most reliable "green" option.

High-pressure hydroelectric power stations require construction of dams and reservoirs, which affects landscape and changes ecological properties of environment [5-7]. But there is an alternative to highpressure stations - free-stream hydropower stations [8], which are described in this paper.

Computational fluid dynamics is the most convenient method for studying behavior of rotating machinery from hydropower turbines to wind turbines [9-11]. This method is also applicable for free-stream hydropower stations. Most of CFD papers aims to describe one or several working regimes 12-13]. This leads to stationary methods usage and doesn't allow to take transitional effects into consideration. On the other hand, unstationary methods require more attention for problem set-up [14] and more computational resources.

\section{Methods}

Consider acceleration of free-stream hydropower turbine wheel under influence of hydrodynamic and inertia forces. Let's assume wheel as system with one rotating degree of freedom, implying that displacements on others degrees of freedom are negligible. This approach is used in papers before [15-17]

Free-stream turbine's blade system was designed in ANSYS DesignModeler. Meridian section, leading and trailing edges position were chosen according to flow parameters and wheel diameter. Blade leading edge angle and trailing edge angle were built according to non-separated flow condition and necessary swirl value. Blade was designed with 3 streamlines (close to hub, close to shroud and mid-point between hub and shroud). Angle variation principle for each streamline was chosen based on smoothness condition. Blade thickness was chosen according to material durability. Hub was designed to reach non-separated flow (fig. 1). Wheel diameter is $270 \mathrm{~mm}$.

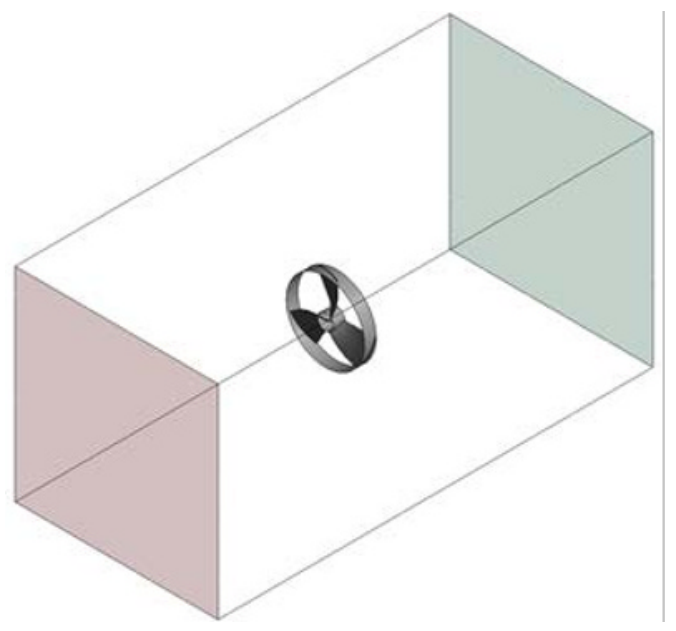

Fig. 1. Computational domain with the free-stream turbine wheel.

\footnotetext{
* Corresponding author: svyatoslav.khorobrov@gmail.com
} 
One can integrate pressure and friction on wheel surface to get hydrodynamic force FL. Flow acts on wheel with force FL and moment ML, causing wheel rotation. Wheel's momentum of inertia I0 were obtained from CAD “Kompas 3D” after completing wheel desing and choosing materials. Wheel motion can be described by simple law:

$$
\begin{aligned}
& I_{0} \beta=\sum M ; \beta=\dot{\omega}=\frac{d \omega}{d t} ; \\
& \sum M=M_{L} \rightarrow I_{0} \frac{d \omega}{d t}=M_{L}
\end{aligned}
$$

One can obtain difference formula for wheel angular velocity which depends on flow parameters (that's possible because the momentum of inertia is constant for constant geometry; $\mathrm{n}-1$ and $\mathrm{n}$ denotes to previous and current timesteps):

$$
\begin{gathered}
\text { first order }: \omega_{n}=\Delta t \frac{M_{n}}{I_{0}} \\
\text { second order }: \omega_{n}=\omega_{n-1}+\Delta t \frac{3 M_{n}-M_{n-1}}{2 I_{0}}
\end{gathered}
$$

This FSI-method was described in [15]. It allows to obtain wheel motion parameters without external load.

From now on let's apply load on wheel. Generator with known characteristics $\mathrm{N}(\omega)$ was chosen as load. One can get the momentum of load from characteristics using simple formula $\mathrm{N}=\mathrm{M} \omega$, so we obtain $\mathrm{Me}$ (omega) dependence. Now we can describe wheel motion as:

$$
I_{0} \frac{d \omega}{d t}=M_{L}-M_{E}
$$

Where Me is generator load. In differential form we will need to change expression for $\mathrm{Mn}$ :

$$
M_{n}=M_{n L}-M_{E}\left(\omega_{n-1}\right)
$$

Now we have the similar differential formula for angular velocity. And we will need one extra condition: generator momentum is less or equal than wheel momentum because generator can't take more power than turbine wheel can provide.

Navier-Stokes equations employed to model incompressible flow around wheel without external forces applied. Fluid density is $998.2 \mathrm{~kg} / \mathrm{m}^{3}$, fluid viscosity is $0.001003 \mathrm{~Pa} * \mathrm{~s}$. Inlet flow velocity is $2.5 \mathrm{~m} / \mathrm{s}$ so Reynolds number of the flow is $6 * 105$. Reynolds number is quite high, so we can assume flow fully turbulent. We transformed Navier-Stokes equations to RANS and completed the system with k-w SST turbulence model according to [18]

Computational domain merged into 2 subdomains one static subdomain and one rotating subdomain (volume around the wheel). Second subdomain rotates simultaneously with wheel. Boundary conditions defined as follows: constant velocity set at inlet (green area, look fig.1), constant (atmospheric) static pressure set at outlet (red area), non-slip wall condition set on wheel surfaces and symmetry set on the rest of boundaries. This setup allows to reduce size of the computational domain. Initial condition formed from stationary computation with same boundary conditions.

RANS equations solved using finite-volume method and ANSYS Fluent solver. Unstructured mesh boult for finite-volume computations. Each wall surface had an inflation layer that was designed to resolve turbulent boundary layer. User-defined function employed to calculate angular velocity of free-stream turbine wheel.

\section{Results and discussion}

Firstly, we will analyze the computation of wheel motion without external load. We aim at velocity field change over time and overall wheel characteristics change over time (hydrodynamic force momentum on wheel, wheel power, wheel angular velocity)

Both momentum and power grow upon exact moment (for momentum this moment is $0.32 \mathrm{~s}$, for power this moment is $0.47 \mathrm{~s}$ ), then both fall down to almost zero values. Angular velocity grows from zero to constant value. Constant value for angular velocity obtained at $1.5 \mathrm{~s}$.

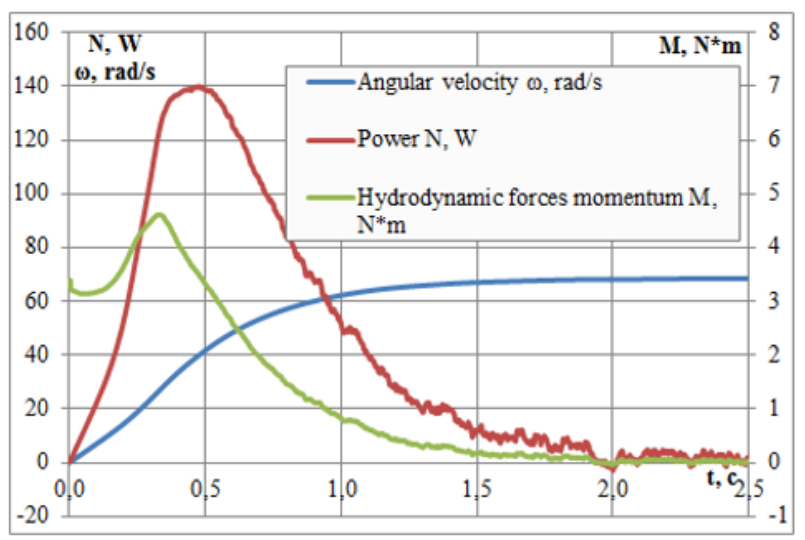

Fig. 2. The distribution of integral characteristics over time.

Velocity fields at longitudinal cross-section obtained for momentum rise, momentum peak, power peak, momentum decrease and after 1.5 s (look at fig. 3-5). 


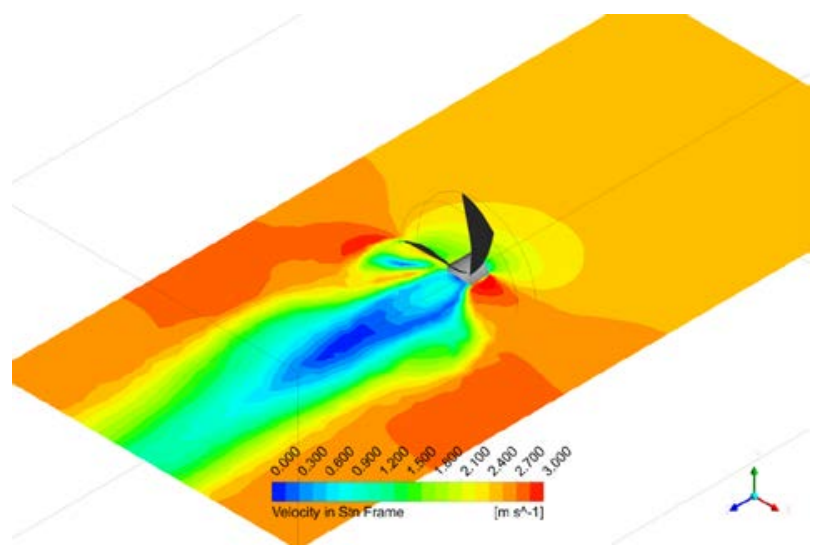

Fig. 3. Velocity field in the longitudinal section during the growth of momentum and power.

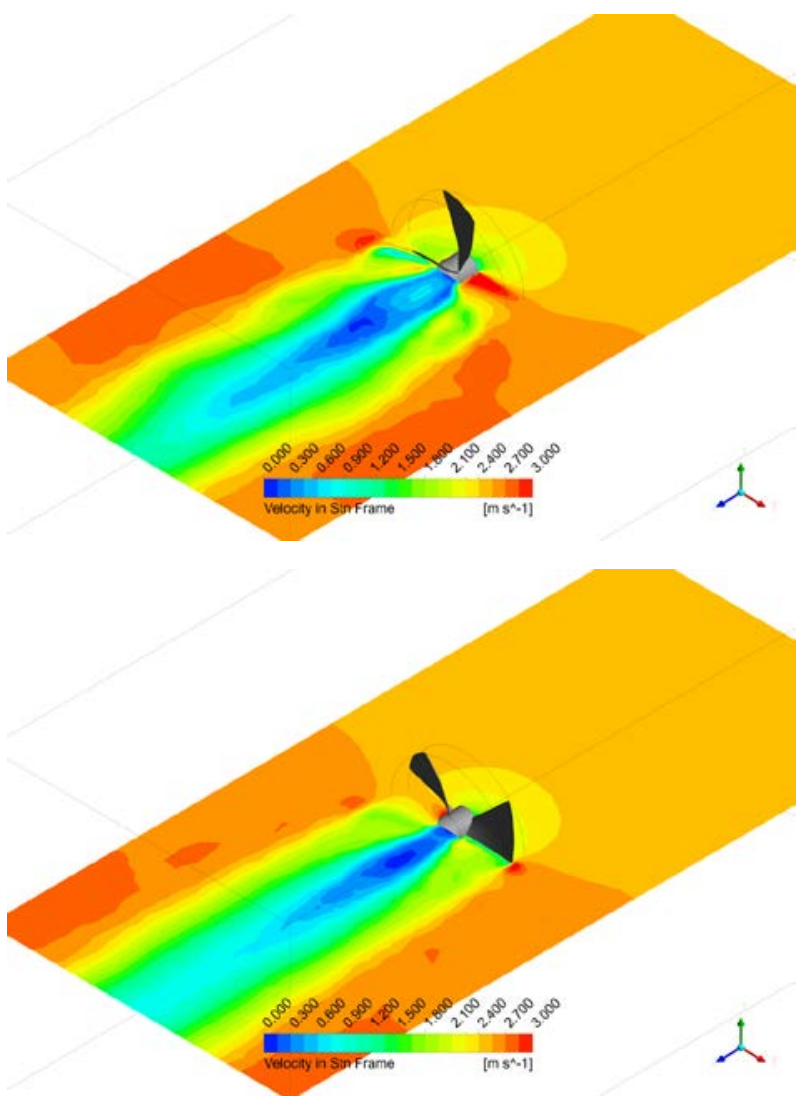

Fig. 4. Velocity field in the longitudinal section during the peak of momentum (upper figure) and power (lower figure).
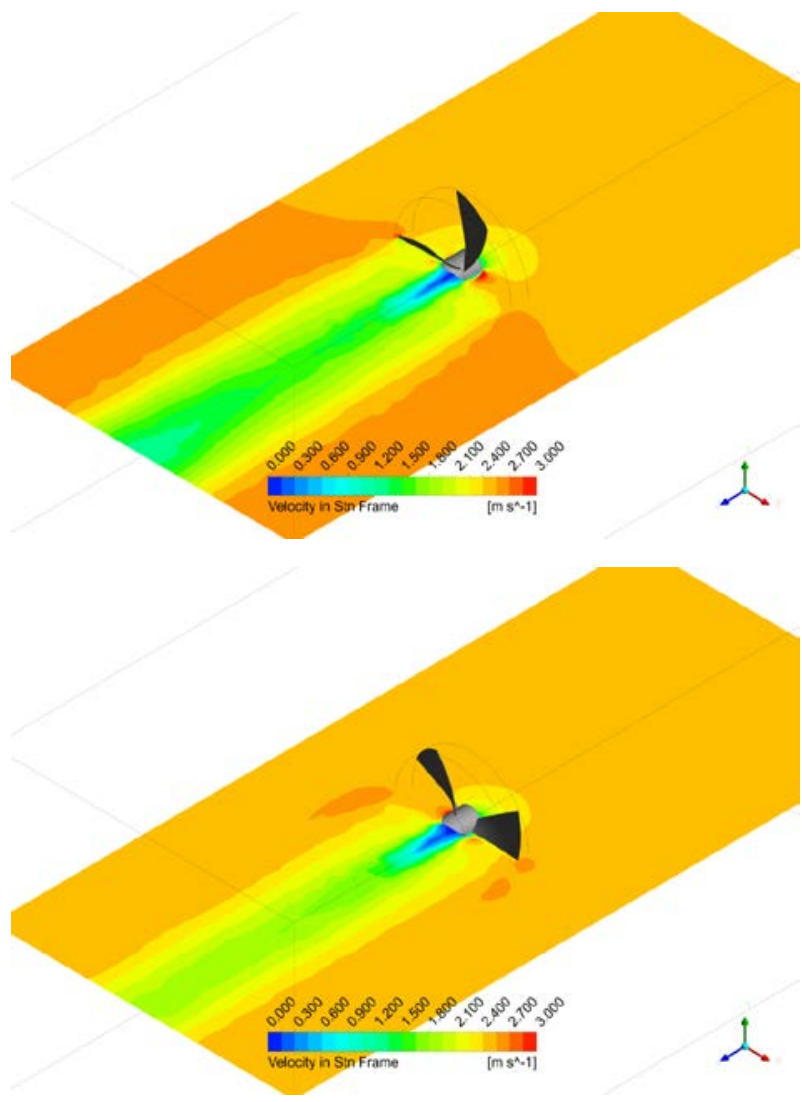

Fig. 5. Velocity field in the longitudinal section during the drop in momentum and power (upper figure) and steady wheel motion (lower figure).

At the beginning of wheel motion, (look at fig. 3) velocity field is completely asymmetric both on peripheral zone and in the wheel wake. As angular velocity increase, flow uniformity begins to rise (fig. 4, 5). At first peripheral zone become more stable, then wheel wake size decreases. Size of the zone with lowest velocity (the so-called drag zone) also decreases with rise of angular velocity.

High angular velocity causes more fluid involved into motion. This effect increases flow stability. Angular velocity of wheel change velocity distribution downstream the wheel slightly. On the other hand, velocity distribution upstream the turbine wheel is very sensitive to angular velocity value.

Next assumption is that generator load applied on wheel. Both wheel and generator are located on one shaft, so angular velocities are equal. Comparison of wheel behavior with and without generator load (look at fig. 6) leads to several conclusions. When load is applied, moment on wheel decreases faster because of wheel deceleration by generator, also angular velocity of wheel increases slower. Stationary regime obtained at lower angular velocity. Power on turbine wheel under load is decently smaller in compare with "clean” wheel. This explained by momentum (and power) take-off from wheel to generator. Besides, in case of applied load wheel power does not characterize full power of system as it was in case of no load applied.

Full power of the system is sum of applied load power and wheel power. 


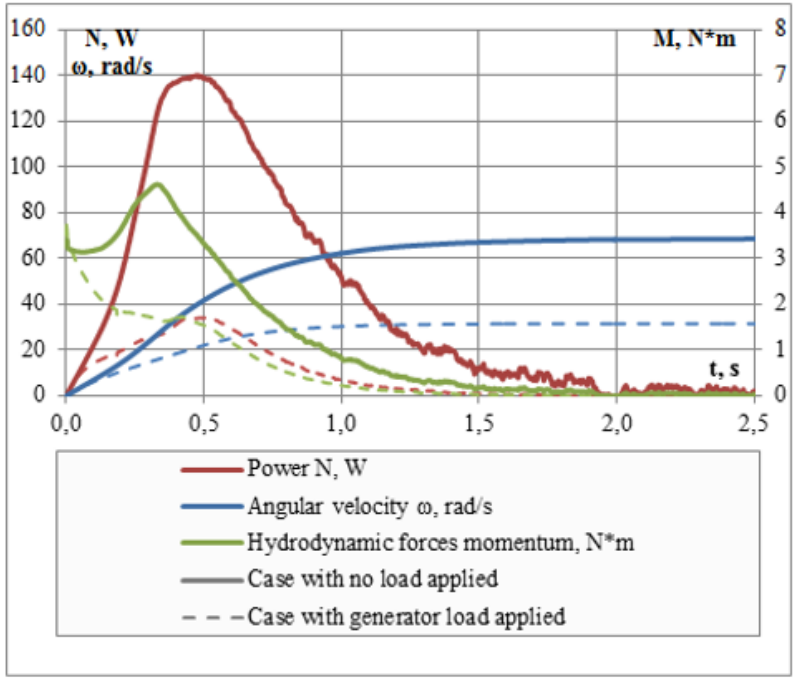

Fig. 6. The distribution of integral characteristics over time for cases with and without generator load.

Consider dimensionless angular velocity change. Dimensionless angular velocity $\mathrm{w}$ can be defined as follows:

$$
\omega^{\prime}=\omega / \omega_{\text {STATIONARY }}
$$

Where w_stat is stationary angular velocity that correspond to constant regime. W' can easily describe difference in angular acceleration (look at fig. 7). One may notice than in case of the generator load applied velocity growth rate is bigger than in case of external load absence.

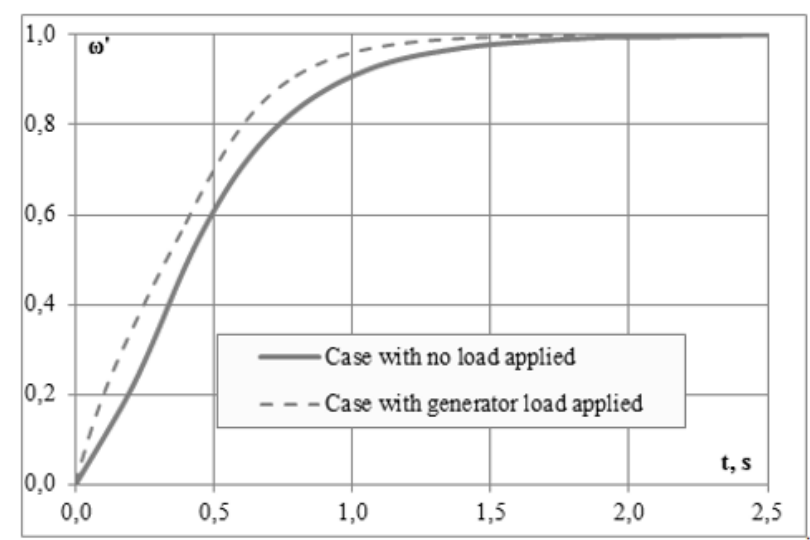

Fig. 7. The distribution of dimensionless angular velocity over time for cases with and without generator load.

\section{Conclusion}

Free-stream turbine startup simulated using RANS finite volume method along with SST turbulence model and one-way FSI approach. Simulation has been held with external load (generator) and without external load. Difference between these two regimes has been shown. Also, the importance of considering startup period have been shown.

\section{References}

1. Borovkov, A.I., Voinov, I.B., Galerkin, Y.B., Drozdov, A.A., Soldatova, K. V. Experimental characteristic simulation for two-stage pipeline centrifugal compressor. IOP Conference Series: Materials Science and Engineering. 2019. 604(1). DOI:10.1088/1757899X/604/1/012052.

2. Stepanov, O.A., Rydalina, N. V., Antonova, E.O., Aksenov, B.G., Derevianko, O. V., Akhmetova, I.G., Zunino, P. The possibility of increasing the operating efficiency of gas turbines at compressor stations of main gas pipelines. International Journal of Civil Engineering and Technology. 2019. 10(2). Pp. 2130-2137.

3. Rekstin, A., Semenovskiy, V., Soldatova, K., Galerkin, Y., Sokolov, K. The simulation of gas-dynamic characteristics of centrifugal compressors in turbo-expander units. E3S Web of Conferences. 2019. DOI:10.1051/e3sconf/201912401008.

4. Analysis of the indicators of the balances of electric energy and power of the UES of Russia for the 1st quarter of 2019. Moscow, 2019.

5. Zhirnov, A.E., Skrebneva, E.V. The environmental impact of hydropower plants. ENERGOSTART conference proceedings. Kemerovo, 2016.

6. Brashkova, A.N. The influence of high-pressure power plants on the hydrological regime of the Yenisei River. Materials of the scientific-practical school-seminar of young scientists and specialists in the field of hydrometeorology. Novosibirsk, 2012.

7. Korpachev, V.P. Problems of predicting pollution and clogging with wood pulp and organic matter in highpressure hydroelectric reservoirs. The successes of modern natural history. 2004. 2. Pp. 110-113.

8. Kazhinsky, B.B. Low power free-flow hydroelectric power stations. Moscow, "State Energy Publisher", 1950.

9. Troldborg, N., Zahle, F., Sørensen, N.N. Simulation of a MW rotor equipped with vortex generators using CFD and an actuator shape model. 53rd AIAA Aerospace Sciences Meeting. 2015. DOI:10.2514/6.2015-1035.

10. Karthikeyan, T., Ezhilsabareesh, K., Samad, A., Venkatesan, N., Avital, E.J. Parametric analysis of a tidal current turbine using CFD techniques. Progress in Renewable Energies Offshore - Proceedings of 2nd International Conference on Renewable Energies Offshore, RENEW 2016. 2016. Pp. 553-558. DOI:10.1201/9781315229256-66.

11. Itou, T., Karasawa, R., Yoshino, M. CFD Contribution to Development ofSmall Wind Turbine Generator. NTN TECHNICAL REVIEW. 2016. No.84. Pp. 34-39.

12. Alaimo, A., Esposito, A., Messineo, A., Orlando, C., Tumino, D. 3D CFD analysis of a vertical axis wind turbine. Energies. 2015. 8(4). Pp. 3013-3033. DOI:10.3390/en8043013.

13. Dewan, Y., Custer, C., Ivashchenko, A. Simulation of the Francis-99 Hydro Turbine during Steady and Transient Operation. Journal of Physics: Conference Series. 2017. 782(1). DOI:10.1088/1742-6596/782/1/012003.

14. Decaix, J., Hasmatuchi, V., Titzschkau, M., MünchAlligné, C. CFD investigation of a high head Francis turbine at Speed No-Load Using advanced URANS models. Applied Sciences (Switzerland). 2018. 8(12). DOI:10.3390/app8122505. 
15. Nicolle, J., Giroux, A.M., Morissette, J.F. CFD configurations for hydraulic turbine startup. IOP Conference Series: Earth and Environmental Science. 2014. 22. DOI:10.1088/1755-1315/22/3/032021.

16. Akinyemi, O.S., Liu, Y. CFD modeling and simulation of a hydropower system in generating clean electricity from water flow. International Journal of Energy and Environmental Engineering. 2015. 6(4). Pp. 357-366. DOI:10.1007/s40095-015-0180-2.

17. Fleisinger, M., Vesenjak, M., Hriberšek, M. Flow driven analysis of a darrieus water turbine. Strojniski Vestnik/Journal of Mechanical Engineering. 2014. 60(12). Pp. 769-776. DOI:10.5545/sv-jme.2014.1712.

18. Menter, F.R. Best Practice : Scale-Resolving Simulations in ANSYS CFD. (April)2012. 